\title{
Modeling and Control Strategies for a Variable Reluctance Direct-Drive Motor
}

\author{
Fabio Filicori, Corrado Guarino Lo Bianco, Alberto Tonielli
}

\begin{abstract}
In industrial automation and robotic applications, direct-drive motors represent a suitable solution to friction and backlash problems typical of mechanical reduction gears. Variable reluctance (VR) motors are well suited for direct-drive implementation but, because of the strongly nonlinear electromechanical characteristics, these motors are traditionally designed as stepper motors.

The main aim of the work described in the paper is the design of a high-performance ripple-free dynamic torque controller for a VR motor, intended for trajectory tracking in robotic applications. An original modeling approach is investigated in order to simplify the design of the high-performance torque controller. Model structure and parameter estimation techniques are presented. Different approaches to the overall torque controller design problem are also discussed and the solution adopted is illustrated. A cascade controller structure is considered. It consists of a feedforward nonlinear torque compensator, cascaded to a nonlinear flux or current closed-loop controller. The feed forward compensator is carefully considered and optimization techniques are used for its design. Two optimization criteria are proposed: the first minimizes copper losses, whereas the second minimizes the maximum value of the motor-feeding voltage. Although developed for a specific commercial motor, the proposed modeling and optimization strategies can be used for other VR motors with magnetically decoupled phases, both rotating and linear. Laboratory experiments for model validation and preliminary simulation results of the overall torque contro system are presented.
\end{abstract}

\section{INTRODUCTION}

$\mathrm{E}$ LECTRICAL motors generally produce their maximum power at very high velocity. When the number of poles is increased, it is possible to obtain higher torque and lower velocity at constant power. Since increasing the number of poles has practical size and cost limitations, a mechanical reduction-gear is typically used. In robotic applications, very low velocity, very high torque, and good accuracy in an angular position represent typical operating specifications. Friction and backlash deriving from mechanical reduction gear impose severe limitations on

Manuscript received August 1, 1991. This work was partially supported by A.X.I.S. s.p.a.-Tavarnelle Val di Pesa (Firenze), M.U.R.S.T.-Progetto Speciale Nazionale "Controllo dei Processi:" CIOC-CNR.

F. Filicori is with the Faculty of Engineering, University of Ferrara, 44100 Ferrara, Italy.

C. G. Lo Bianco and A. Tonielli are with the Department of Electronics, Computer and System Science (DEIS), University of Bologna, 40136 Bologna, Italy. A. Tonielli is the author to whom correspondence should be addressed.

IEEE Log Number 9204611 the performance of positioning servos [1], [2]. Direct-drive connection is the best solution in this application environment.

Direct-drive motors can be obtained by optimizing the design and control of standard dc and ac machines [3] or specifically designing a variable reluctance (VR) [4]-[6] or switched reluctance (SR) [7] motor. A VR motor with a very high number of poles can be designed. Hence, by means of a proper electromechanical configuration it is possible to select the desired torque/velocity ratio.

The ideal robotic actuator, besides direct-drive connection, should reach position with high accuracy, provide constant torque output for every angular position/velocity, and keep constant velocity even in the presence of variable loads. With VR motors, stepping drives [8] are not able to satisfy any of these specifications, mainly because they do not consider motor nonlinearities. Dynamical performance can be improved by feedback control, as shown in [9], but neglected nonlinearities cause torque ripple, thus preventing the use of this approach where accurate trajectory tracking is needed. Torque nonlinearities are considered in [10], where an interesting controller structure is suggested. The limitations of this approach are related to the constant velocity and magnetic linearity assumptions. To meet the specifications required for a robotic actuator, it is necessary to carefully take into account all the motor nonlinearities through a suitable motor model and then design an advanced control system that linearizes motor and load characteristics [11], [12].

Our work aims at the design of a high-performance ripple-free dynamic torque controller for a VR motor, intended for trajectory tracking in robotic applications. To this aim, an accurate non-linear dynamical model of the motor is the basis for any further activity. An original modeling approach is proposed, in order to simplify the design of the high-performance torque controller. The use of flux as the selected state variable plays a fundamental role in the simplification of modeling and control problems. A simple model, which is linear with respect to the control variables, results from this choice; furthermore, unlike other proposed models [5], [11], [12], only nonlinear functions of one independent variable are involved, even when magnetic saturation is considered.

A cascade controller structure similar to the one proposed in [10] was selected. It consists of a static feedforward nonlinear compensator, cascaded to a nonlinear flux 
or current closed-loop controller. The feedforward nonlinear compensator transforms the torque set point into a corresponding flux or current one, whereas the internal (flux or current) closed-loop nonlinear controller is based on estimated or directly measured feedback.

The selection of flux or current as the directly controlled variable in the internal closed-loop controller, depends on different factors [17]. Flux can be the best choice in a microprocessor-based realization, allowing for implementation of a flux observer. Current may be preferred in a sliding mode design, implemented with analog technologies, because it is directly measurable. According to the selection of flux as the state variable in the model, the design of a feedforward compensator is performed with respect to the flux controller. Transformation of a flux set-point into a current one can be easily effected using the proposed model. A current controller is considered in this paper.

In the design of the feedforward compensator, the problem of transforming a scalar torque into an equivalent three-phase flux vector is considered. The proposed model, with its simple structure and nonlinear functions of one variable, greatly simplifies the task.

Unlike [10], optimization techniques are used for the design of the feedforward precompensator, and the nonlinear closed-loop controller is designed in a stator reference frame, thus avoiding the use of coordinate transformations. Two different performance indexes are considered in the optimization procedure; the first, optimal at low velocity, minimizes copper losses, whereas the other, optimal at high velocity, minimizes the inverter voltage required to impress the flux.

Even if it is specifically designed for a commercial VR motor called NSK Motornetics RS-1410 [6], the proposed solution can be used for other VR motors with magnetically decoupled phases, both rotating [4] and linear [5].

In the paper, an original procedure for the determination of a VR motor dynamical model is proposed and compared with other solutions. Model structure and parameter estimation techniques are presented. Different approaches to the overall torque controller design problem are then discussed and the adopted solutions illustrated. Details on the optimal design of the feedforward torque compensator are presented. Two different performance indexes are considered and optimal solutions are compared. Experimental results are then presented to validate the model, whereas the optimized flux profile and the validity of the overall approach are verified by preliminary simulations of the torque control system.

\section{NOMENCLATURE}

$v, i, \Phi \quad$ Voltage, current, flux linkage

$r \quad$ Winding resistance

$T \quad$ Torque; $\tilde{T}$ denotes a particular torque value

$j \quad$ Phase index

$\theta, \omega \quad$ Rotor angular position and velocity

$D, J_{m} \quad$ Rotor dumping factor and inertia

$W^{m} \quad$ Energy stored in the magnetic field
$M \quad$ Number of teeth in the rotor

$N \quad$ Number of turns of a stator winding

\section{Dynamical Model of a Variable Reluctance MOTOR}

The actuator considered in this work is the NSKMotornetics RS-1410 three-phase rotating motor, whose structure is shown in Fig. 1. The most interesting characteristics are the structure of the teeth, the phase positioning, and the double stator. Selection of a structure with a very high number of teeth (150) corresponds to the choice of a direct-drive realization with very high torque and very low velocity, as required in robotic applications. Phase positioning and double stator guarantee optimized magnetic paths to the flux. For the motor considered, the following properties have been experimentally verified:

- Magnetic hysteresis and Foucault current are negligible

- The three phases are almost completely magnetically decoupled.

Equations describing the stator magnetic circuit are, independently for the three phases,

$$
\frac{d \Phi_{j}(t)}{d t}=-r_{j} i_{j}(t)+v_{j}(t) \quad j=0,1,2 .
$$

In the following, whenever this does not generate misunderstandings, references to phase and time will be omitted. In order to put (1) in a state space form, two different alternatives exist, depending on the selected state variable.

The current, being directly measurable, is the most common and natural choice in a model built for control purposes. This solution, adopted in [5] and [11], leads to the following nonlinear model:

$$
\begin{array}{r}
\frac{d i}{d t}=-\left(\frac{\partial \Phi(i, \theta)}{\partial i}\right)^{-1}\left(r i+\frac{\partial \Phi(i, \theta)}{\partial \theta} \omega\right) \\
+\left(\frac{\partial \Phi(i, \theta)}{\partial i}\right)^{-1} v
\end{array}
$$

with nonlinearities that are functions of two variables.

Alternatively, the flux can be selected as the state variable, leading to the following nonlinear model:

$$
\frac{d \Phi}{d t}=-r f(\theta, \Phi)+v
$$

This simpler formulation, when saturation of magnetic circuits is being considered, still requires the definition of a nonlinear function of two variables and is based on a state variable that is not directly measurable. In our research activity model (3) is considered. This paper shows how the specific nonlinear function in two variables $f(\theta, \Phi)$ contained in (3) can be split into the sum of two nonlinear functions in one variable and how this model can simplify the design of a ripple-free dynamic torque controller. 


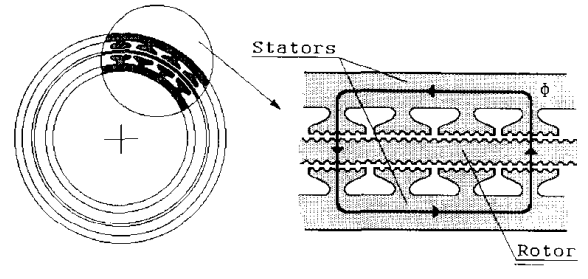

Fig. 1. Cross-section of the motor and magnetic flux path of a phase.

In order to obtain the complete state-space dynamic model for the motor, the nonlinear function $f(\theta, \Phi)$ in (3) must be specified, as well as torque and mechanical equations. As far as the $f(\theta, \Phi)$ function is concerned, a nonlinear periodic dependence on the angular position must be considered for a VR motor. On the other hand, the flux can enter linearly or nonlinearly depending on motor operating conditions. A linear magnetic circuit can be considered, greatly simplifying the model [10], [14] However, in several application fields-as, for example, robotics - high accelerations are required and the motor must be operated under magnetic saturation conditions to maximize the torque/mass ratio. This imposes considering also a nonlinear dependence of function $f(\theta, \Phi)$ on the flux.

The procedure for the determination of $f(\theta, \Phi)$ starts by considering the magnetomotive force (m.m.f.) developed by a single phase:

$$
N i=H(\theta, \Phi)
$$

On analyzing the magnetic circuit shown in Fig. 1, it can be assumed that the m.m.f. is decomposable in two components: the first, which refers to the iron portion of the magnetic flux path, nonlinearly depends on flux and is assumed to be almost independent on the angular position; the second, taking into account the air-gap portion, is assumed to be a linear function of flux and periodically depends on the angular position. Including the scaling factor $N$ in the nonlinear functions, an expression for the current is, therefore,

$$
i=F(\Phi)+R(\theta) * \Phi
$$

where $F(\Phi)$ describes nonlinear effects in the iron part of the flux path, whereas $R(\theta)$ represents a position-dependent term associated to the air-gap reluctance. In particular, $R(\theta)$ can be interpreted as a normalized air-gap reluctance.

The torque equation can be derived in several different ways [15], [16]. For example, by means of the D'Alembert principle, assuming a virtual displacement at constant flux, the motor torque generated by a single phase is

$$
T=-\frac{\partial W}{\partial \theta}=-\int_{0}^{\Phi} \frac{\partial i\left(\theta, \Phi^{*}\right)}{\partial \theta} d \Phi^{*}
$$

From (5), it follows that

$$
T=-\int_{0}^{\Phi} \frac{\Phi^{*} d R(\theta)}{d \theta} d \Phi^{*}=-\frac{\Phi^{2}}{2} \frac{d R(\theta)}{d \theta} \triangleq \Phi^{2} h(\theta) .
$$

Equation (7) quantifies two well-known properties of VR motors. The first property is that torque is a quadratic function of flux. This means that the sign of the torque developed by a single phase does not depend on the sign of the flux (and hence of the current) but on the motor angular position only. The second property is that at constant flux, the torque is proportional to the derivative of the air-gap reluctance with respect to position. This explains why a motor with a larger number of teeth must be designed in order to generate a larger torque. Besides, (7) shows that to generate a constant torque independently of the angular position, a variable flux must be generated since $R$ is a periodic function of angular position.

Considering also mechanical equations, the complete dynamical model for the motor is:

\section{A. State Equations}

$$
\begin{gathered}
\frac{d \Phi_{j}}{d t}=-r f_{j}\left(\theta, \Phi_{j}\right)+v_{j} ; j=0,1,2 \\
\frac{d \omega}{d t}=-\frac{D}{J_{m}} \omega-\frac{1}{2 J_{m}} \sum_{j=0}^{2} \frac{d R_{j}(\theta)}{d \theta} \Phi_{j}^{2} \\
\frac{d \theta}{d t}=\omega
\end{gathered}
$$

\section{B. Output Equations}

$$
\begin{gathered}
i_{j}=f_{j}\left(\theta, \Phi_{j}\right)=F\left(\Phi_{j}\right)+R_{j}(\theta) \Phi_{j} ; j=0,1,2 \\
T=-\frac{1}{2} \sum_{j=0}^{2} \frac{d R_{j}(\theta)}{d \theta} \Phi_{j}^{2} .
\end{gathered}
$$

\section{Model Identification and Parameter ESTIMATION}

In the previous paragraph, the structure of a model for VR motors was introduced. Two different parameter estimation techniques are proposed. The first requires current and flux measurements only, whereas the second also needs the torque. In the final paragraph reporting experimental results, it is shown that a direct flux measurement is not strictly required, since flux can be obtained indirectly by current and feeding voltage measurements.

The only terms to be identified in (8)-(11), because they are not directly available from motor data sheets, are $F(\Phi)$ and $R(\theta)$. Before we enter into estimation details, it is worth remembering that, due to the complete symmetry of the three phases of the motor, the unknown functions must be defined for a single phase only. Hence, to simplify notations, reference to the phase will be omitted. 


\section{A. Estimation Method Based on Current and Flux Measurement}

Remembering that $F(\Phi)$ represents nonlinearities related to magnetic saturation, a monotonic function can be assumed to be of the kind

$$
F(\Phi)=\sum_{r=1}^{H} F_{r} \Phi^{r}=F_{1} \Phi+F^{*}(\Phi)
$$

where $F_{r}$ represents the coefficients of the expansion and $F^{*}$ is a strictly nonlinear function since it includes all the polynomial terms of an order greater than one. The term $F_{0}$ has been neglected because magnetic hysteresis is negligible. Consequently, (5) can be rewritten as:

$i=F^{*}(\Phi)+\left(F_{1}+R(\theta)\right) \Phi=F^{*}(\Phi)+R^{\circ}(\theta) \Phi$.

Determination of $R^{\circ}(\theta)$ : Since $F^{*}(\Phi)$ is a strictly nonlinear function, it follows from (13) that

$$
i \approx R^{\circ}(\theta) \Phi \quad \text { if } \Phi \Rightarrow 0
$$

Term $R^{\circ}(\theta)$ represents the slope of the curve $i(\theta, \Phi)$ around the operating point $\Phi+0$ and, hence,

$$
\frac{1}{R^{\circ}(\theta)}=\left.\frac{\partial \Phi(\theta, i)}{\partial i}\right|_{i \approx 0}
$$

Samples of $R^{\circ}(\theta)$ at a different angular position $\theta_{n}$ can be experimentally derived by estimating the slope of the corresponding flux curve, as a function of the current, near the origin. To completely define function $R^{\circ}(\theta)$, a suitable interpolating function and an approximation criterion must be defined. By defining $\theta=0$ as the position of full stator and rotor teeth alignment for the phase considered, observing that $R^{\circ}(\theta)$ is a periodic function symmetrical with respect to $\theta=0$, the following representation can be selected:

$$
R^{\circ}(\theta)=R_{0}+R(\theta)=R_{0}+\sum_{r=1}^{L} R_{r} \cos (M r \theta) .
$$

Order $L$ and coefficients $R_{r}$, defining the trigonometric series (16), can be computed by means of discrete Fourier transform.

Determination of $F^{*}(\Phi)$ : Equation (13) gives

$$
F^{*}(\Phi)=i-R^{\circ}(\theta) \Phi
$$

From any $\theta$, (e.g., $\theta=0$ ), samples of $F^{*}(\Phi)$ can be obtained from (17) (after the computation of $R^{\circ}(\theta)$ ) by means of experiments at different current and flux.

According to (12), order $H$ and coefficients $F_{r}$, completely defining the polynomial function $F^{*}(\Phi)$, can be obtained by means of the least-squares method.

\section{B. Estimation Method Based on Current, Flux, and Torque Measurements}

Bearing in mind (16), torque equation (7) can be rewritten as

$$
\frac{d R(\theta)}{d \theta}=\frac{d R^{\circ}(\theta)}{d \theta}=-\frac{2 T}{\Phi^{2}} .
$$

Values of $d R(\theta) / d \theta$ (or $d R^{\circ}(\theta) / d \theta$ ), sampled at different rotor positions $\theta_{n}$, can be obtained by means of flux and torque measurements. Using (16) for $R^{\circ}(\theta)$, it is possible to obtain

$$
\frac{d R^{\circ}(\theta)}{d \theta}=-\sum_{r=1}^{L} R_{r} M r \sin (\operatorname{Mr} \theta) .
$$

Again, order $L$ and coefficients $R_{r}$ of (19) can be calculated by means of the discrete Fourier transform. Coefficients $R_{r}$ also parametrize the periodical function $R^{\circ}(\theta)$ in (16) up to constant $R_{0}$. If $R_{0}$ is considered equal to zero, an estimate of function $R(\theta)$ is obtained. This is not a limitation because the constant term $R_{0}$ can be considered to be the $F_{1}$ term of $F(\Phi)$ function, (see (12) and (13)).

The procedure proposed in Section III-A for the estimation of $F^{*}(\Phi)$ is not limited by the hypothesis that the function is strictly nonlinear. This observation enable the same procedure to be used for the estimation of function $F(\Phi)$ in the most general form (12).

\section{General Structure of a Nonlinear Torque CONTROLlER}

In motor equations (8)-(11), strong non-linearities are present. The design of a closed-loop controller for a VR motor is hence quite difficult and requires a precise definition of the control targets as well as advanced control algorithms.

In this paper interest is concentrated on robotic applications. The multiple-axis robot controller is usually designed under the assumption that torque actuators are available. Electric motors are not directly torque actuators; a suitable drive configuration is required. Standard $\mathrm{dc}$ or ac drives are usually designed as velocity or position actuators even if they internally contain current loops. In robotic applications a torque drive could be preferable, whereas velocity and position control of coordinate axes are dealt with in the main robot controller. These considerations have suggested the design of a torque-controlled drive for the VR motor. For this particular type of motor, besides imposing a desired dynamic behavior on the torque, the controller should also guarantee ripple-free torque operation independently of rotor position and velocity.

Direct implementation of a closed-loop torque controller would require torque measurement or estimation. This solution is, in general, very expensive and not convenient in a direct-drive application, because a torque sensor between the motor and the load would introduce undesired elasticities in the link. On the other hand, strong nonlinearities and very low motor velocity prevent simple implementation of a motor torque observer. 


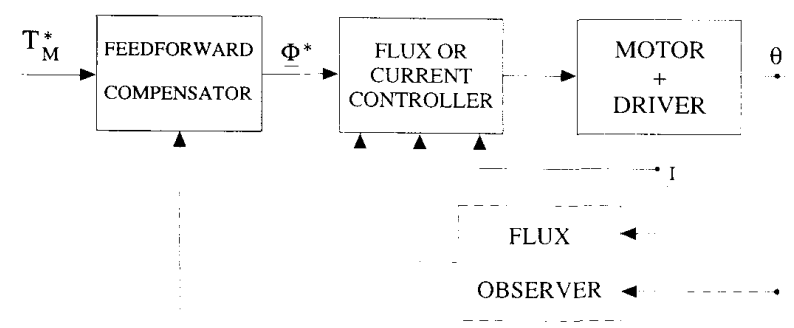

Fig. 2. Block diagram of the controller.

In motor drives based on standard $\mathrm{dc}$ and ac motors (both synchronous and asynchronous), the torque control problem can be solved by transforming it into an equivalent current control one. This simple solution is possible because the torque is proportional to the current, or to a specific component of the current vector in a proper reference system. Besides, owing to the wide availability of high-quality and low-cost current transducers, this solution is also convenient from an economical point of view.

For VR motors, the torque versus current function is nonlinear, thus preventing the simple solution adopted in drives for standard motors. To overcome this problem a cascade controller structure, similar to the one proposed in [10] and illustrated in Fig. 2, has been selected. It consists of an external static feedforward nonlinear compensator, followed by a nonlinear flux or current (depending on design choices) closed-loop controller. The feedforward compensator transforms the torque set point into a corresponding flux or current one, whereas the internal closed-loop controller is based on estimated or directly measured feedback, depending on the controlled variable selected. Unlike [10], optimization techniques are used for the design of a feedforward precompensator, and the closed-loop controller operates in a stator reference frame, thus avoiding the use of coordinate transformations.

This paper presents motor modeling and control optimization activities. Emphasis is placed on the optimization techniques used in the design of the feedforward compensator. Work related to the design of the closedloop flux or current controller is currently in progress [17] and is only briefly reported here in order to validate the design of the feedforward compensator. Before entering into details about the feedforward compensator design, some general considerations are worth making.

Direct calculation of a current set point is not convenient because torque dependence on current must also consider magnetic nonlinearities. Recalling (6), a simpler relationship exists between torque and flux. Therefore, the feedforward compensator is designed under the assumption of an internal flux closed-loop controller. If current is selected, the flux set point can be directly converted into a current one by means of the model output equation (11a). It must be pointed out how the proposed model structure greatly simplifies the design of the torque controller.
A critical point is the transformation of the scalar torque request into a corresponding three-phase flux vector. Recalling motor equation (1), it can be noted that fluxes relative to different phases can be impressed independently by means of the associated control inputs $v_{j}$; the control problem thus has as many degrees of freedom as the number of phases. These degrees of freedom can be used for different purposes. In [10], for a four-phase motor, two adjacent phases, selected according to the actual rotor position and torque sign, are used to impose torque dynamics and ripple-free operation. The remaining two phases are controlled in order to keep their current at zero. In [11], for an $m$-phases motor, the desired dynamics is imposed on motor acceleration by controlling a single phase, selected as a function of position and torque sign. The remaining controls must keep the remaining phase currents at zero or take them to zero as fast as possible.

Both approaches have problems, mainly related to the need for a fast switch-on and switch-off of phase currents that impose a voltage waveform that is strongly impulsive. Since the voltage is limited in a real power inverter, an increase in the residual torque ripple occurs. Furthermore, the solution proposed in [11], while allowing good dynamic specification of the error between the actual and the desired acceleration, does not control the torque ripple explicitly.

The approach considered in this paper attempts to use as many degrees of freedom as possible in order to get the best performance from the motor. As already mentioned, the selected controller structure is shown in Fig. 2. Indeterminations in transforming the scalar torque $T_{M}^{*}$ into the flux vector $\phi^{*}$, are used inside the feedforward compensator to obtain optimal ripple-free "torque-sharing" flux functions. Two optimization criteria are proposed: the first, optimal at low velocity, minimizes copper losses; the second, optimal at high velocity, minimizes the maximum value of the motor feeding voltage at maximum velocity and torque.

Independently of the selected optimization criterion, due to motor symmetry, "torque-sharing" flux functions are periodical and the same function is optimal for all three phases. For every rotor position $\theta$, the same function can be used for the three phases with an argument $\theta_{j}=\theta+j \theta_{s}(j=0,1,2)$ shifted by one-third or two-thirds of a motor step $\left(\theta_{s}=\frac{1}{3} *(2 \pi / M)\right)$. Moreover, optimal "torque-sharing" flux functions can be computed off-line, in the worst operating condition, for a single torque value (typically, a large torque value $\bar{T}$ deliverable without ripple).

In fact, from the optimal flux function $\tilde{\Phi}(\theta)$ computed at the maximum torque $\tilde{T}$ deliverable without ripple under the specific operating conditions, the flux function corresponding to a lower torque value $T=\tilde{T} K(0 \leq K \leq 1)$ can be obtained by

$$
\Phi(\theta)=\tilde{\Phi}(\theta) \sqrt{K}
$$

By means of this simple scaling operation, suboptimal 
functions, ensuring ripple-free torque operation, can be easily computed on line by the controller. Suboptimality of the computed flux functions can be easily tolerated because, with both the selected optimization criteria, operations at lower torque surely correspond to relaxed operating conditions. Because paper attention is concentrated on motor modeling and feedforward compensator optimization, details on the closed-loop flux or current controller [17] are omitted.

\section{A. Compensator Minimizing Copper Losses}

With reference to Fig. 3, it can be noted that one or two phases, depending on the rotor position, generates a torque of a given sign. Determination of ripple-free "torque-sharing" flux functions minimizing copper losses $\left(P_{c}\right)$ implies sharing torque only between phases giving torque in the desired direction.

Within subintervals where only a single phase generates torque of a given sign, no optimization is required, and determination of flux function can be effected directly by means of (7):

$$
\Phi(\theta)=\sqrt{\frac{T}{h(\theta)}} .
$$

Within subintervals where two phases generate torque of a given sign (e.g., for positive torque, phases 0 and 2 in the subinterval $\theta_{3}<\theta<\theta_{4}$ of Fig. 3), a problem of the kind

$$
\begin{aligned}
\Phi^{2}(\theta) h(\theta)+\Phi^{2}\left(\theta+2 \theta_{s}\right) h\left(\theta+2 \theta_{s}\right) & =\bar{T} \\
& \theta_{3}<\theta<\theta_{4}
\end{aligned}
$$

must be solved. Problem (22) has infinite solutions; a particular solution can be obtained by means of optimization techniques.

If the selected optimization criterion is minimization of copper losses $\left(P_{c}\right)$, the feedforward compensator can be designed by solving the optimization problem $\min _{\Phi(\theta)}\left\{P_{c}\right\}$, with the constraint of constant torque in the subinterval.

More specifically, if phases 0 and 2 are still considered (see (11a)), the problem becomes

$$
\begin{array}{r}
\min _{\Phi(\theta)}\left\{f^{2}[\theta, \Phi(\theta)]+f^{2}\left[\left(\theta+2 \theta_{s}\right), \Phi\left(\theta+2 \theta_{s}\right)\right]\right\} \\
\theta_{3}<\theta<\theta_{4}
\end{array}
$$

with constraint (22). By using constraint equation (22), the following relation can be easily obtained:

$$
\Phi\left(\theta+2 \theta_{s}\right)=\sqrt{\frac{\tilde{T}-\Phi^{2}(\theta) h(\theta)}{h\left(\theta+2 \theta_{s}\right)}} \triangleq g(\theta, \Phi(\theta)) .
$$

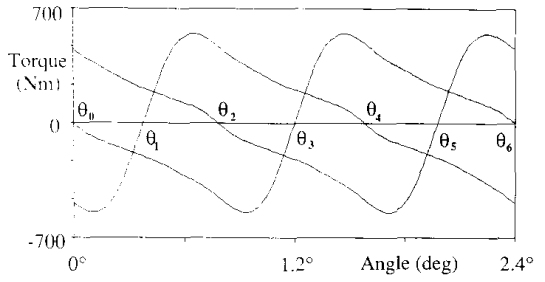

Fig. 3. Torque curves generated by the three phases at constant flux.

By substituting (24) into (23), the original constrained minimization problems (23) and (22) are equivalent to the following unconstrained one:

$$
\begin{array}{r}
\min _{\Phi(\theta)}\left\{f^{2}[\theta, \Phi(\theta)]+f^{2}\left[\left(\theta+2 \theta_{s}\right), g(\theta, \Phi(\theta))\right]\right\} \\
\theta_{3}<\theta<\theta_{4}
\end{array}
$$

After discretization, the solution can be obtained using a standard numerical algorithm for the minimization of a nonlinear function. The periodical function $\Phi(\theta)$ reported in Fig. 4, is derived by means of a polynomial expansion in the period.

Analysis of the optimal flux profile shown in Fig. 4 presents regions with very high slope. According to (1), a motor feeding voltage increasing with velocity results. Numerical values show that at angular velocities larger than $0.2-0.3 \mathrm{r} / \mathrm{s}$ (corresponding to $20-30 \%$ of the maximum value), the motor-feeding voltage required to impose the flux in these regions is larger than the maximum voltage admissible for the motor. This stimulates the search for other optimization criteria that are more suitable for positioning transients at high velocity.

\section{B. Compensator Minimizing Motor-Feeding Voltage}

To overcome acceleration limitations at high velocity, due to power inverter voltage saturation, the optimal flux function can be computed by means of a different optimization procedure.

Optimal flux is a function of position and torque. Hence,

$$
\Phi=\Phi(\theta, T)
$$

Neglecting the ohmic voltage drop, it follows that the feeding voltage required to impose this flux is

$$
V=\frac{d \Phi}{d t}=\frac{\partial \Phi}{\partial \theta} \frac{d \theta}{d t}+\frac{\partial \Phi}{\partial T} \frac{d T}{d t}
$$

To limit voltage requirements, a flux function minimizing (27) must be computed. Constrained minimization of (27) would require an a priori knowledge of torque and its time derivative. In a real situation, the torque controller 


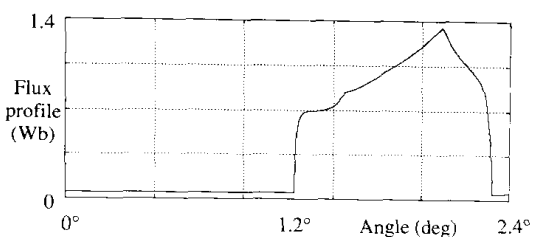

Fig. 4. Flux profile for each phase, optimal at low velocity (computed for $T=170 \mathrm{Nm}$ )

operates in a cascade structure with a velocity/position external controller that dynamically changes its instantaneous torque request. An a priori knowledge of torque and torque derivative cannot be assumed. On the other hand, the bandwidth of the external velocity/position controller can be considered to be slower than that of position-dependent flux (especially at high motor velocity), and the following simplification can be made:

$$
V \cong \frac{\partial \Phi}{\partial \theta} \omega
$$

Moreover, if according to the considerations leading to (20), optimization is carried out considering the maximum torque $\tilde{T}$, the optimal flux function depends only on rotor position $\theta$.

The problem to be solved is thus

$$
\min _{\Phi(\theta)}\left\{\max _{\theta}\{|V(\theta)|\}\right\}
$$

with the constraints

$$
\left\{\begin{array}{l}
0 \leq \Phi(\theta) \leq \Phi_{\max } \\
\sum_{j=0}^{2} \Phi^{2}\left(\theta+j \theta_{s}\right) h\left(\theta+j \theta_{s}\right)=\tilde{T} .
\end{array}\right.
$$

This is a min/max optimization problem with a cost function containing the derivative with respect to position of the unknown quantity and subject to linear as well as nonlinear (quadratic) constraints.

To solve the problem, discretization of continuous functions with respect to rotor position is performed. Let us define with $S$ the number of samples contained within a motor step. Therefore, the width of a sampling interval is $\Delta=(2 \pi / M) / S$; the generic position displacement within a step is $\theta_{n}=n^{*} \Delta, n=0,1, \cdots,(S-1)$; and the shift between two adjacent phases is $S^{*} \Delta / 3=k \Delta$.

To simplify notations, reference to $\Delta$ will be omitted and the values of a generic function sampled for $\theta=\theta_{n}$ will be denoted in such a way that, for example, $h\left(\theta_{n}\right)$ will be $h(n), \Phi\left(\theta_{n}\right)$ will be $\Phi(n)$ and so on. With the symbols thus defined, the torque developed by the motor in the generic position $\theta_{n}$, is

$$
T(n)=\sum_{j=0}^{2} T_{j}(n+j k)=\sum_{j=0}^{2} \Phi_{j}^{2}(n+j k) h(n+j k) .
$$

Remembering that the unknown function $\Phi(\theta)$ is a periodic function, it is well known that a linear matrix operator exists such that

$$
\Phi^{\prime}=\mathbf{D} \Phi
$$

where $\mathbf{D} \in R^{S * S}$ is the linear matrix operator and

$$
\begin{gathered}
\boldsymbol{\Phi}=[\Phi(0), \Phi(1), \cdots, \Phi(S-1)]^{T} \\
\boldsymbol{\Phi}^{\prime}=\left.\frac{\partial \Phi}{\partial \theta}\right|_{0 \leq n<S-1}=\left[\Phi^{\prime}(0), \Phi^{\prime}(1), \cdots, \Phi^{\prime}(S-1)\right]^{T} .
\end{gathered}
$$

By virtue of (28) and (32), the optimization problem (29), (30), can be rewritten as

$$
\min _{\mathbf{\Phi}}\left\{\|\omega \mathbf{D} \boldsymbol{\Phi}\|_{\infty}\right\}
$$

with constraints

$$
\begin{aligned}
0 \leq \Phi(n) & \leq \Phi_{\max } \\
\sum_{j=0}^{2} \Phi^{2}(n+j k) h(n+j k) & =\tilde{T} \quad 0 \leq n<S .
\end{aligned}
$$

In (33), $\|\mathbf{x}\|_{\infty}$ denotes the $\infty$ norm of the vector $\mathbf{x} \in R^{N}$, defined as

$$
\|\mathbf{x}\|_{\infty}=\lim _{p \rightarrow \infty}\left(\sum_{l=1}^{N}\left|x_{1}\right|^{p}\right)^{1 / p}
$$

Remembering that the $\infty$ norm of a vector defines the maximum of the absolute value of the elements of the vector, an auxiliary variable $E$ can be introduced, representing the maximum value of the inverter voltage required to impose the optimal flux as

$$
E=\max _{n}\left\{\omega \mathbf{D}_{n} \boldsymbol{\Phi},-\omega \mathbf{D}_{n} \boldsymbol{\Phi}\right\}
$$

where $\mathbf{D}_{n}$ denotes the $n$th row of $\mathbf{D}$.

Problems (33) and (34) can finally be reformulated as

$$
\min _{\boldsymbol{\Phi}}\{E\}
$$

with original constraints (34) and the added constraints

$$
\left\{\begin{array}{l}
E \geq+\omega \mathbf{D}_{n} \mathbf{\Phi} \\
E \geq-\omega \mathbf{D}_{n} \boldsymbol{\Phi}
\end{array} \quad n=0,1, \cdots, S-1 .\right.
$$

The original min-max problem has thus been transformed into the equivalent linear minimization problem (35), with linear (36) (34a) and quadratic (34b) constraints.

For the solution of this problem, a recursive algorithm based on a linear programming kernel (Simplex) [18] is used, under the assumption that $\omega$ is a known constant (e.g., $\left.\omega=\omega_{\max }\right)$. At the generic iteration, the Simplex method is applied to a problem obtained by linearization of nonlinear constraints (34b) around the previous solution. The algorithm proceeds until the "current solution" 


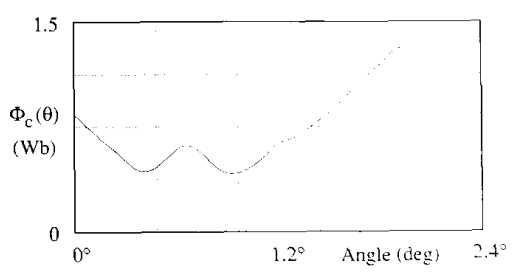

Fig. 5. Flux profile for each phase, optimal at high velocity (computed for $T=170 \mathrm{Nm}$ )

is equal to the previous one. To ensure reliable convergence, further constraints must be added to limit the maximum admissible displacement of the "current solution" from the previous one.

The optimal solution is reported in Fig. 5. According to simplifications made to define the optimization problem, under dynamical operating conditions higher feeding voltage must be expected. Some preliminary simulation experiments of the torque control system showed feeding voltages to be only slightly larger, thus fully justifying the validity of the approximations made.

An important observation is that the optimal flux profile never goes to zero. This means that a single phase generates flux also when it produces a negative torque, thus slightly reducing the maximum value of torque that can be theoretically impressed without ripple at the maximum velocity. This is not a real limitation, however, because without the optimal profile, it would be impossible to get that torque without ripple because of feeding voltage limitations.

\section{EXPERIMENTAL VALIDATION OF THE MODEL AND} PRELIMINARY SimUlations OF TORQUE CONTROL

In Section III, algorithms for identification have been proposed. Measurement experiments as well as computational procedures and experimental results are now given in some details.

The availability of flux measurements is assumed for the determination of the functions $R(\theta)$ and $F(\Phi)$. This would require fiux sensor inside the motor. It is shown how it is possible to avoid direct flux measurements by using current and voltage measurement only.

Remembering (1),

$$
\Phi=K+\int_{0}^{t}(v-r i) d t .
$$

Constant $K$ is selected in order to obtain a flux curve that is symmetric with respect to zero. It is possible to obtain the flux from voltage and current curves. Accuracy of the method obviously depends on the accuracy of the measurements and knowledge of the motor resistance. A brief outline of the experiments performed to obtain the model parameters using the second method based also on torque measurement is given next.

First is the determination of the flux as a function of current and position. With the rotor blocked in a precise position, a periodical voltage is applied to a phase of the motor, while voltage and current are recorded. Numerical integration of (37) led to the solution. The same procedure is then repeated for different rotor positions. The experimental flux curve shown in Fig. 6 presents a very low hysteresis, thus confirming validity of the assumption made at the beginning of the paper, and lack of saturation when teeth are completely unaligned.

Second is the determination of $d R(\theta) / d \theta$ by means of (18). With the motor in the same positions already used to calculate the flux, a prescribed current is imposed on the phase and the torque is measured. From the flux curve reported in Fig. 6 the corresponding flux is then calculated. The experiments showed a slight dependence on the current (and hence on the flux) of $d R(\theta) / d \theta$. In the least-squares computation of the coefficients in expansion (19), a redundant number of samples must be used, taken at different values of the current. The slight dependence on the current is thus considered as a disturbance.

Third is the determination of $F(\Phi)$ from (17). As in the previous case, a slight dependence on the rotor position has been noticed. Also in this case, the samples for the least-squares computation of the coefficients in (12) must be selected larger than strictly required by using measurements taken at different angular positions.

In Figs. 7 and 8, experimental curves are reported for $R(\theta), d R(\theta) / d t$, and $F(\Phi)$, respectively. The results refer to the method reported in Section III-B, that considers coefficient $R_{0}$ in (16) to be equal to zero. This implies that $R(\theta)$ assumes both positive and negative values. The other estimation method proposed in Section III-A would lead to a coefficient $R_{0}$ different from zero or, equivalently, to a strictly positive reluctance function $R^{\circ}(\theta)$.

The model derived has been tested against the actual motor comparing the estimated and measured values for different variables and operating conditions. The first comparison is related to the torque. Measured and estimated values at different positions and currents are reported in Fig. 9. Good local and global agreement is shown. The mean square error is $2.3 \%$ at $5 \mathrm{~A}$ and $7.8 \%$ at $7.5 \mathrm{~A}$, for a total of $6.1 \%$ at all different currents.

The second comparison is related to the current. Measured and estimated values with $20 \mathrm{~Hz}$ sinusoidal feeding voltage at different positions are reported in Fig. 10. Very good accuracy is shown even for $\theta=0$ when magnetic saturation strongly influences the circuit. The global (for all positions) mean-square error is $4.2 \%$.

Proposed flux optimization strategies are then compared in terms of flux derivative normalized with respect to angular velocity, (proportional to feeding voltage), and dissipated power. In Fig. 11 normalized flux derivatives are reported, showing critical regions for the first optimal function (i.e., the one minimizing losses). Twice per period, the feeding voltage required to impose that flux derivative becomes very high. The second optimization procedure (minimizing feeding voltage) obviously gives a smoother behavior leading to lower voltage requirements. On the other hand, Fig. 12 shows a larger power dissipation associated to the second optimal profile; the average 


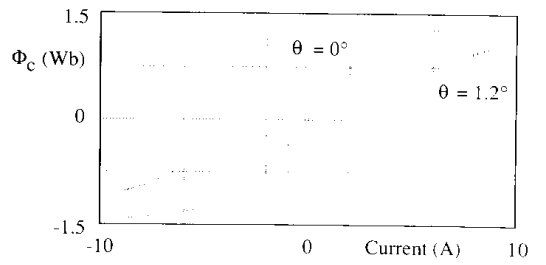

Fig. 6. Flux as a function of current at different positions. $(\theta=0$ corresponds to full teeth alignment).

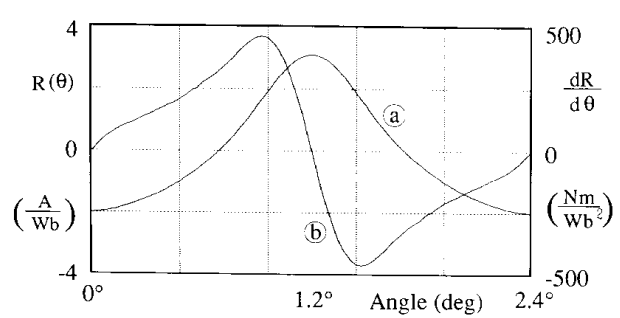

Fig. 7. Periodic functions $R(\theta)$ (a) and $d R(\theta) / d t$ (b) in a motor step.

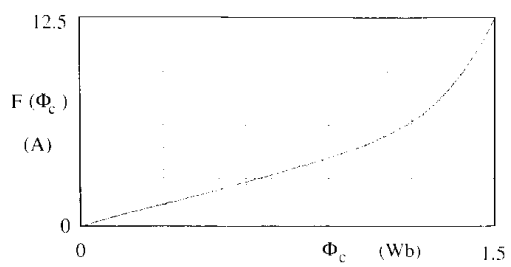

Fig. 8. Function $F(\Phi)$. Dots around the curve represent experimental values.

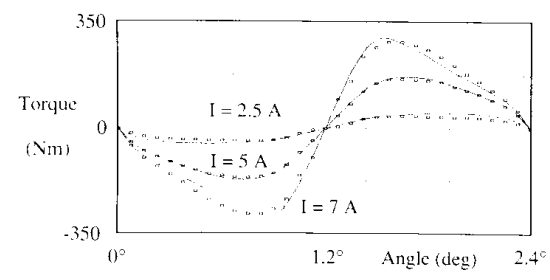

Fig. 9. Measured and estimated (solid line) torque at different current values in a motor step.

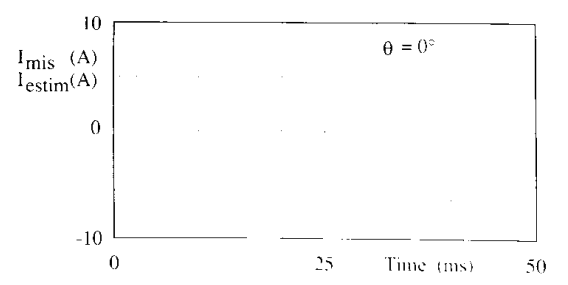

Fig. 10. Measured and estimated current with $20 \mathrm{~Hz}$ sinusoidal feeding voltage at $\theta=0$; magnetic circuit saturates.

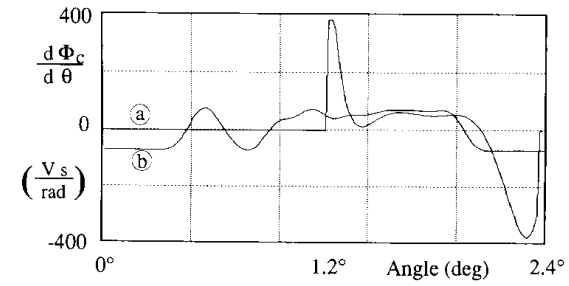

Fig. 11. Normalized flux derivative (proportional to motor feeding voltage) for the two flux optimization methods. (a) Minimization of copper losses. (b) Minimization of maximum feeding voltage.

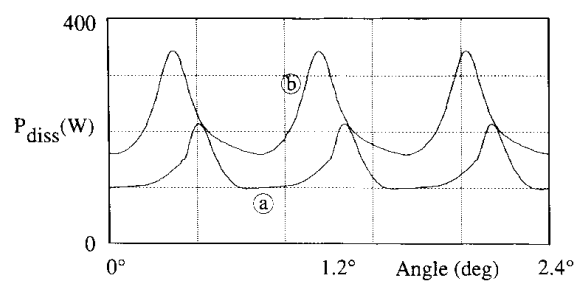

Fig. 12. Dissipated power for the two flux optimization methods. (a) Minimization of copper losses. (b) Minimization of maximum feeding voltage.

increase is in the order of 1.7 times. Increase in power dissipation is due partly to the different profile and partly to the fact that the three phases are always fed, even when they give a negative torque. Investigations are being carried out to define torque ripple-free flux functions depending on motor velocity, thus reducing losses at low velocity and feeding voltage at high velocity.

To complete the presentation, some preliminary simulations-made with the MICOSS Simulation Package [19] and related to torque control-are reported in Fig. 13. In the simulated controller, optimal flux profiles are converted into current ones by means of model (11a). A current sliding mode controller is used because of its robustness properties and very low computational requirements [17], [20], [21].

A very low high-frequency torque ripple (lower than $7 \%$ peak to peak), partially due to some residual control delay, demonstrates the validity of the approach adopted. Reduced on-line computational requirements, commutation frequencies lower than $25 \mathrm{khz}$, and voltage and current levels well inside standard values all confirm complete feasibility of a ripple-free torque controller for the VR direct-drive motor.

\section{CONClusions AND Future DEVElopment}

The definition of a specific motor model, suitable for control system design, as well as the use of advanced optimization algorithms for flux profile determination are the main steps for the design of a VR motor ripple-free torque control.

The original modeling approach that uses fluxes as state variables and permits the derivation of the current through 


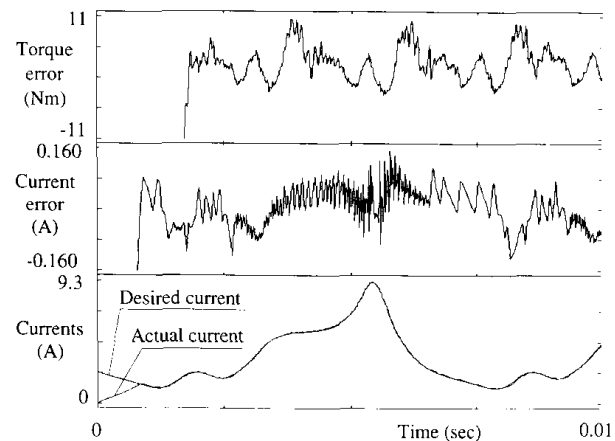

Fig. 13. Simulation results of the sliding mode torque control of the VR motor. (a) Steady-state torque error. (b) Phase 1 current error. (c) Optimal current profile, actual and desired.

a simple two variable equation plays a fundamental role in the proposed technique.

Optimization procedures for determination of constanttorque flux profiles, permits the nonlinear torque-generation mechanism, typical of VR motors, to be easily compensated for. The two proposed methods both have advantages and disadvantages; the first is optimal in terms of power dissipation but suitable for application only at low velocity, whereas the second is optimal in terms of motor feeding voltage but leads to a higher dissipated power. A combined approach is currently under investigation.

Intensive experimental activity has been carried out in order to define a suitable model parametrization procedure. Quite simple analytical expressions for the relevant motor parameters were obtained using the Fourier transform and least-squares methods for parameter fitting.

Research activity is in an advanced development phase relative to design and implementation of a robust velocity/torque controller for the use of VR motors in directdrive robotic applications.

\section{ACKNOWLEDGMENT}

The authors wish to acknowledge the work of students who strongly supported the research activity during and after their degree thesis (Laurea) activity.

\section{REFERENCES}

[1] L. M. Sweet and M. C. Good, "Redefinition of the robot motion control problem: Effects of plant dynamics, drive system constraints and user requirements," in Proc. 23rd Conf. Decision Contr., Las Vegas, NV, Dec. 1984, pp. 724-732.

[2] R. Marino and M. W. Spong, "Nonlinear control techniques for flexible joint manipulators: A single link case study," in Proc. 1986 IEEE Int. Conf. Robotics Automation, San Francisco, CA, April 1986, pp. $1030-1036$.

[3] N. Hemati, J. S. Thorp, and M. C. Leu, "Robust nonlinear control of brushless DC motors for direct-drive robotic applications," IEEE Trans. Ind. Elect., vol. 37, no. 6, pp. 460-468, Dec. 1990.

[4] R. Welburn, "Ultra high torque motor system for direct-drive robotics," Motor Con. Proc., Atlantic City, NJ, April 1984.

[5] D. G. Manzer, M. Varghese, and J. S. Thorp, "Variable Reluctance Motor Characterization," IEEE Trans. Ind. Electron., vol. 36 no. 1, pp. 56-63, Feb. 1989
[6] Motornetics Corporation, "Megatorque Motor System," California, Dec. 1983.

[7] W. F. Ray, P. J. Lawrenson, R. M. Davis, M. Stephenson, N. N. Fulton, and R. J. Blake, "High-performance switched reluctance brushless drives," IEEE Trans. Industry Applications, vol. IA-22, no. 4, pp. $722-729,1986$.

[8] J. Ish-Shalom and D. Manzer, "Commutation and control of step motors," in Proc. 14th Annu. Symp. Incremental Motion Control System Device, B. C. Kuo, Ed. Incremental Motion Control Systems Society, Campaign, 1985, pp. 283-292.

[9] B. K. Bose, T. J. E. Miller, P. M. Szczesny, and W. H. Bicknell, "Microcomputer control of switched reluctance motor," IEEE Trans. Industry Applications, vol. IA-22, no. 4, pp. 708-715, July/Aug. 1986.

[10] M. Ilić-Spong, T. J. E. Miller, S. R. Macminn, and J. S. Thorp, "Istantaneous torque control of electric motor drivers," IEEE Trans. Power Electron., vol, PE-2, no. 1, pp. 55-61, Jan. 1987.

[11] M. Ilić-Spong, R. Marino, S. M. Peresada, and D. G. Taylor, "Feedback linearizing control of switched reluctance motors," IEEE Trans. Automat. Contr., vol. AC-32, no. 5, pp. 371-379, May 1987.

[12] M. Ilić-Spong and F. K. Mak, "Torque control of switched reluctance drives with the saturation included," Incr. Motion Conf., Champaign, IL, 1986, pp. 275-282.

[13] G. S. Buja and M. I. Valla, "Control characteristics of a SRM drive taking into account the motor saturation," in Proc. 16th Annual Conf., IECON '90, Pacific Grove, CA, Nov. 1990, pp. 1013-1021.

[14] A. Lumsdaine and J. H. Lang, "State observer for variable-reluctance motors," IEEE Trans. Ind. Electron., vol. 37, no. 2, pp. 133-142, April 1990.

[15] H. H. Woodson and J. R. Melcher, Electromechanical Dynamics, vol. 1. New York: Wiley, 1968.

[16] A. Fitzgerald, C. Kingsly, and A. Kusko, Electrical Machinery. New York: McGraw-Hill, 1971.

[17] C. Rossi, A. Tonielli, C. Guarino Lo Bianco, and F. Filicori, "Robust control of a direct-drive variable reluctance motor," in Proc. IEEE/RSJ, Int. Workshop on Intelligent Robots and Systems, IROS '91, Nov. 2-5, 1991, Osaka, Japan, pp. 337-343.

[18] C. H. Papadimitriou and K. Steiglitz, Combinatorial Optimization. Englewood Cliffs, NJ: Prentice-Hall, 1982.

[19] C. Bonivento, A. Tonielli, and R. Zanasi, Simulazione di Sistemi Dinamici con programma MICOSS. Bologna, Italy: Pitagora Editrice, 1990 (in Italian).

[20] V. I. Utkin, Sliding-Mode and Their Application in Variable Structure System. Moscow: Mir Publishers, 1978.

[21] C. Rossi and A. Tonielli, "Sliding-mode control of ac motor drives: Analysis of mapping functions properties," in Proc. IEEE Workshop on Intell. Motion Contr., Istanbul, Turkey, Aug. 1990, pp. $749-756$.

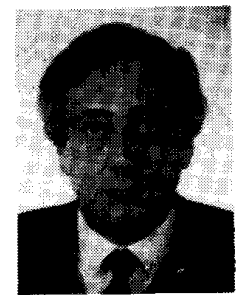

Fabio Filicori was born in Imola, Italy, in 1949. He received the Dr. Ing. degree in electronic engineering at the University of Bologna in 1974. He joined the Department of Electronics of the University of Bologna in 1974 as an Assistant Researcher and later became Associate Professor of Applied Electronics. In November 1990 he became Full Professor of Applied Electronics at the University of Perugia. Last year he joined the Faculty of Engineering of the University of Ferrara, where he is Full Professor responsible for the Electronic Engineering degree course. During his academy career he has held courses on computer-aided circuit design, electronic devices and circuits, and power electronics. His main research activities are in the areas of computer-aided design techniques for nonlinear circuits, power electronics, electrons' device modeling, and electronics measurements. 
